\title{
Ulipristal acetate before high complexity endoscopic (hysteroscopic, laparoscopic) myomectomy - a mini-review
}

\author{
Włodzimierz Baranowski \\ Department of Gynaecological Oncology, St John's Oncological Centre, Lublin, Poland \\ Department of Gynaecology and Gynaecological Oncology, Military Institute of Medicine, Warsaw, Poland
}

\begin{abstract}
Uterine myomas (fibromas, leiomyomas) are the most common tumours in women, and their clinical signs and symptoms are presented by $25-40 \%$ of patients with these benign tumours.

According to current guidelines, the armamentarium for myoma management consists of: medical therapy (GnRH, SPRMs), non-surgical alternatives including uterine artery embolisation (UAE), vaginal temporary occlusion of uterine arteries using clamp-like device or MRgFUS technique, and surgical treatment (including minimally invasive techniques). In cases of submucous myomas STEPW classification correlates very well with the risk of incomplete hysteroscopic myomectomies. According to limited literature data, ulipristal acetate as a pre-treatment seems to be very prudent in high complexity hysteroscopic myomectomy (STEPW II, score 5-6). In patients with large uterine myomas (FIGO type 3, 4, 5) undergoing laparoscopic myomectomy, three-month pre-treatment with ulipristal acetate before laparoscopy is feasible and can be recommended because of shorter time of surgery, lower intraoperative blood loss, lower haemoglobin drop, and low postoperative blood transfusion rate.
\end{abstract}

Key words: myoma, hysteroscopy, laparoscopy, ulipristal acetate.

Uterine myomas (fibroids) are benign tumours coming from smooth muscle cells of the uterine wall. From an epidemiological point of view, uterine myomas are the most common tumours in women, and their clinical signs and symptoms can be found in $25-40 \%$ of patients with fibromas [1]. Clinical manifestations of fibromas usually include different kinds of excessive bleeding (menorrhagia, metrorrhagia), and sometimes fibromas are involved in obstetric disturbances (infertility, complications during pregnancy, labour, and puerperium). Large fibromas can also induce pelvic pain and pelvic pressure symptoms with lower urinary tract symptoms (LUTS) and/or constipation [2].

According to current guidelines, surgical strategies of fibroma treatment are dependent on the patient's age as well as the patient's desire to preserve fertility or to preserve the uterus. Current armamentarium of myoma management consists of medical therapy $(\mathrm{GnRH}$, SPRMs). The latest medical therapy using ulipristal acetate seems to be a very interesting option because of its unique mechanism(s) of action and supposed longterm effects. According to basic molecular data, ulipristal acetate is a specific progesterone receptor modulator doing its work by pathways of progesterone. The main pathway of action involves the receptor mecha- nism because ulipristal acetate has very high affinity to progesterone receptor $A$ and $B$. It means that the action of progesterone receptors after ulipristal acetate binding is modulated in different directions (activation or inhibition) giving, at molecular level, modulation of particular genes expression and is involved in proliferation, differentiation, and apoptosis. Additionally, ulipristal acetate alone, like other steroids, is implicated in rapid membrane-initiated changes, which in turn are involved in cell signalling pathway $[2,3]$. In vivo mechanisms of myoma reduction with ulipristal acetate involve inhibition of proliferation rate, extracellular matrix (ECM) remodelling due to matrix metaloproteinase-2 (MMP-2) expression, and a limited period of cell death [4].

Non-surgical alternatives of uterine myomas treatment include uterine artery embolisation (UAE), vaginal temporary occlusion of uterine arteries using clamplike device or MRgFUS technique, and finally surgical treatment with different types of procedure (total hysterectomy, subtotal hysterectomy, myomectomy) using different kinds of approach (laparotomy, laparoscopy, hysteroscopy for submucous tumours) [2].

A few myoma classifications have been described in the literature. The most popular and very simple ESGE classification of submucous fibromas (three types 
of myomas) was adopted from an original paper by Wamsteker et al. published in 1993 [5]. Lasmar et al. [6] published in 2005 and validated in 2011 [7] a new classification of submucous myomas named STEPW (STEPW = Size, Topography, Extension, Penetration, Wall), which seems to be very useful for the prediction of primary success in hysteroscopic techniques. Munro et al. in 2011 [8] described new general classification of fibroids, subsequently adopted by FIGO (International Federation of Gynaecology and Obstetrics), and including nine types of myomas according to its relation (localisation) to the uterine wall (from 0 - pedunculated intracavitary fibroma to 8 - parasitarius or cervical fibroma and also hybrid types, e.g. 2-5).

In cases of submucous myomas STEPW classification correlates very well with the possibility of risk of incomplete hysteroscopic myomectomies [7]. The real problem in hysteroscopy concerns the localisation and size of myoma - sometimes hysteroscopic myomectomy is extremely difficult with high risk of incomplete myomectomy and also with high risk of other complications (excessive bleeding, fluid overload syndrome, etc.). Recently, Ferrero et al. [9] published an original paper on ulipristal acetate treatment before surgery when expecting high complexity hysteroscopic myomectomy. According to original papers published in 2012 by Donnez et al. [10], ulipristal acetate, a specific progesterone receptor modulator (SPRM), is feasible for the management of fibromas before planned surgery. Indeed, nearly half of the patients included in this trial had surgery, but none of the patients had hysteroscopy, so the data on pre-treatment using ulipristal acetate before hysteroscopic myomectomy were very limited. Only Bizzarri et al. [11] in 2015 published the results of a pilot study on pre-treatment before hysteroscopy using triptorelin, letrozole, and ulipristal acetate in patients presenting submucous fibromas, but the number of patients was very limited. Currently Ferrero et al. [9] conducted a retrospective comparative study preoperatively using ulipristal acetate $(5 \mathrm{mg} / \mathrm{d}$ during a consecutive three months) in 25 premenopausal patients with submucous fibroma. The comparative group consisted of similar patients undergoing hysteroscopy without any preoperative treatment. According to STEPW classification only patients scoring 5-6 points were included into the study. The only difference found between two groups was the endometrial thickness, which was nearly two times higher $(8.7 \mathrm{~mm}$ vs. $4.7 \mathrm{~mm}$ in the primary surgery group, $p<0.001)$. The primary endpoint of the trial was to assess the incidence of incomplete resection in the group undergoing ulipristal acetate pre-treatment when compared to the group with primary surgery. Of course, many others outcomes were additionally assessed (fluid absorption, length of surgery, surgical complication e.g. uterine wall perforation, patient satisfaction). In the ulipristal acetate group the changes in myoma size and volume by ultrasonographic examination and adverse effects of drug were also assessed. According to the results obtained by Ferrero et al. [9] it seems that three months of pretreatment with ulipristal acetate in patients presenting submucous myomas with high complexity (STEPW group II, score 5-6) and undergoing complex hysteroscopic procedure, significantly increases the chances of complete primary resection. Additionally, in the group with ulipristal acetate, the time of surgery was significantly lower when compare to the group undergoing direct surgery (28.6 minutes vs. 37.4 minutes, $p<0.05$ ). Recently, Sancho et al. [12] published results of a comparative study on three-month pre-treatment with ulipristal acetate or $\mathrm{GnRH}$ analogues before planned hysteroscopy and found no difference between the two regimes in terms of primary complete resection, but in the surgeon's subjective opinion, ulipristal acetate pre-treatment was associated with an easier resection. Specific endometrial changes described in literature as PAEC (progesterone-receptor modulator-associated endometrial changes) had no adverse impact on surgery [13]. Safety and feasibility seemed to be comparable to hysteroscopic myomectomies with pre-treatment with $\mathrm{GnRH}$ analogues, but with fewer side effects in the group on ulipristal acetate. At present, it seems to be too early to confidently recommend the use ulipristal acetate pre-treatment in all patients with submucous fibroma undergoing hysteroscopy, because many more well-conducted trials are needed.

A very similar conclusion can be drawn from literature data on gonadotropin-releasing hormone analogues - a study performed by Muzii et al. [14] showed reduction in operative time and lower fluid absorption, and the overall difficulty of hysteroscopy was significantly reduced. Contrary to the paper by Muzzi et al. [14], the results of a British double-blind, placebo controlled, randomised trial [15] including patients with very similar profile do not support the routine $\mathrm{GnRH}$ analogue pre-treatment before planned hysteroscopy. Finally, based on systematic literature review and meta-analyses, there is no clear evidence that pre-treatment with gonadotropin-releasing hormone analogues should be routinely used before hysteroscopic myomectomy. Accordingly, the conclusion drawn by Ferrero et al. [9] on selective use of ulipristal acetate as a pre-treatment in clearly recognised and defined clinical situations (STEPW II, score 5-6) seems to be very prudent.

Laparoscopic myomectomy is a much more challenging procedure when compared to hysteroscopic myomectomy, independently from progress made in laparoscopic technology (new multifunctional instruments, 3D-imaging, robotic surgery). Surprisingly, the search of literature data on pre-treatment with ulipristal acetate before laparoscopic myomectomy revealed only one case report. Also, in an original paper by Don- 
nez et al. [10] nearly half of the patients had surgery after treatment, but there was no detailed description of the approach method of myomectomy performed (laparoscopic or laparotomy). The only paper recently published by Ferrero et al. [16] described a group of 34 patients undergoing laparoscopic myomectomy with planned pre-treatment with ulipristal acetate ( $5 \mathrm{mg} /$ day, three-month continuous therapy). As a control group, 43 patients with direct laparoscopic surgery were analysed. According to FIGO classification, only patients having type 3,4 , or 5 myomas were analysed. Only patients with the largest fibroma more than $10 \mathrm{~cm}$ in diameter were included in this study; the total number of myomas was no more than three, the largest diameter of the second myoma was no more than $5 \mathrm{~cm}$, and the third fibroma no more than $3 \mathrm{~cm}$. Retrospective analysis of intra- and postoperative parameters clearly indicates that the intraoperative blood loss was lower in the group pre-treated with ulipristal acetate $(507 \mathrm{ml}$ vs. $684 \mathrm{ml}$ in control, $p=0.012$ ); however, in Table 3 in the original paper [16] there are quite different numbers. Also, the total time of surgery was significantly lower in the ulipristal acetate group (137.6 minutes vs. 159.7 minuets, $p<0.001$ ); however, there was no significant difference in the suturing time between study groups (35 minutes vs. 29 minutes in control, $p=0.076$ ). According to blood loss data, it is obvious that the haemoglobin drop was lower in the ulipristal acetate group (1.1 $\pm 0.5 \mathrm{~g} / \mathrm{dl}$ vs. $1.3 \pm 0.7 \mathrm{~g} / \mathrm{dl}$ in control, $p=0.034$ ). Because the haemoglobin level was higher in patients undergoing pre-treatment with ulipristal acetate, no patient in the group on ulipristal acetate required postoperative blood transfusions, whereas six patients in the control group did $(p=0.031)$. The number of other complications was not different between pre-treated and control groups $(p=0.726)$.

The main conclusions drawn from this study is that routine three-month pre-treatment with ulipristal acetate before laparoscopy in patients with large uterine myomas is feasible and should be recommended because it decreases the length of surgery, intraoperative blood loss, haemoglobin level drop, and consequently postoperative blood transfusion rate.

\section{Disclosure}

Supported by Gedeon Richter.

Author reports no conflict of interest.

\section{References}

1. Lumsden MA, Hamoodi I, Gupta J, Hickey M. Fibroids: diagnosis and treatment. BMJ 2015; 351: h4887.

2. Donnez J, Dolmans MM. Uterine fibroid management: from the present to the future. Hum Reprod Update 2016; 22: 665-686.

3. Moravek M, Yin P, Ono M, et al. Ovarian steroids, stem cells and uterine leiomyoma: therapeutic implications. Hum Reprod Update 2015; 21: 1-12.
4. Courtoy GE, Donnez J, Marbaix E, Dolmans MM. In vivo mechanisms of uterine myoma volume reduction with ulipristal acetate treatment. Fertil Steril 2015; 104: 426-434.

5. Wamsteker K, Emanuel MH, de Kruif JH. Transcervical hysteroscopic resection of submucous fibroids for abnormal uterine bleeding: results regarding the degree of intramural extension. Obstet Gynecol 1993; 82: 736-740.

6. Lasmar RB, Barrozo PR, Dias R, Oliveira MA. Submucous myomas: a new presurgical classification to evaluate the viability of hysteroscopic surgical treatment-preliminary report. J Minim Invasive Gynecol 2005; 12 308-311.

7. Lasmar RB, Xinmei Z, Indman PD, et al. Feasibility of a new system of classification of submucous myomas: a multicenter study. Fertil Steril 2011; 95: 2073-2077.

8. Munro MG, Critchley HO, Broder MS, Fraser IS; FIGO Working Group on Menstrual Disorders. FIGO classification system (PALM-COEIN) for causes of abnormal uterine bleeding in nongravid women of reproductive age. Int J Gynaecol Obstet 2011; 113: 3-13.

9. Ferrero S, Racca A, Tafi E, et al. Ulipristal acetate before high complexity hysteroscopic myomectomy: A retrospective comparative study. J Minim Invasive Gynecol 2016; 23: 390-395.

10. Donnez J, Tatarchuk TF, Bouchard P, et al. PEARL I Study Group. Ulipristal acetate versus placebo for fibroid treatment before surgery. N Engl J Med 2012; 366: 409-420.

11. Bizzarri N, Ghirardi V, Remorgida V, et al. Three-month treatment with triptorelin, letrozole and ulipristal acetate before hysteroscopic resection of uterine myomas: prospective comparative pilot study. Eur J Obstet Gynecol Reprod Biol 2015; 192: 22-26.

12. Sancho JM, Delgado VS, Valero MJ, et al. Hysteroscopic myomectomy outcomes after 3-month treatment with either ulipristal acetate or GnRH analogues: a retrospective comparative study. Eur J Obstet Gynecol Reprod Biol 2016; 198: 127-130.

13. Bettocchi S, Baranowski WE, Doniec J, et al. Hysteroscopic patterns in women on treatment with ulipristal acetate $5 \mathrm{mg}$ /day: A preliminary study. Minim Invasive Gynecol 2016; 3: 664-669.

14. Muzii L, Boni T, Bellati F, et al. GnRH analogue treatment before hysteroscopic resection of submucous myomas: a prospective, randomized, multicenter study. Fertil Steril 2010; 94: 1496-1499.

15. Mavrelos D, Ben-Nagi J, Davies A, et al. The value of pre-operative treatment with $\mathrm{GnRH}$ analogues in women with submucous fibroids: a double-blind, placebo-controlled randomized trial. Hum Reprod 2010; 25: 2264-2269.

16. Ferrero S, Alessandri F, Vellone VG, et al. Three-month treatment with ulipristal acetate prior to laparoscopic myomectomy of large uterine myomas: a retrospective study. Eur J Obstet Gynecol Reprod Biol 2016; 205: 43-47. 